\title{
Supporting researchers: mentoring and awards of the Journal
}

\author{
Aribert Rothenberger
}

Published online: 7 June 2011

(c) The Author(s) 2011. This article is published with open access at Springerlink.com

For a journal like European Child and Adolescent Psychiatry (ECAP) it is of utmost importance to attract outstanding, clinically relevant research. In order to reach this goal, the Journal's reviewers and editors try to be as constructive and supportive as possible for the authors, especially for those who are not yet fully experienced in conducting a study and writing a paper, i.e. the task of ECAP is also to offer some kind of mentoring. In the long run, this should help to improve the quality of research in child and adolescent psychiatry (CAP) and publications in ECAP. Moreover, ECAP would like to show that many attractive and innovative papers already exist that pave the way to the future of CAP. The authors of this kind of research need to be encouraged to go on with their work and improve it.

The today's issue of ECAP gives some good examples for this attitude.

A neuropsychiatric case report on the behaviour of MECP2 duplication syndrome [1] contrasts with two large-scale family-related epidemiological studies from The Netherlands which inform (a) about the parental behaviour like depression and hostility which are associated with an increased risk of child emotional and behavioural problems already at the age of three years [7] and (b) about the parental psychological control which was strongly related to the anxiety symptoms in adolescents [8]. It is a pity that both the epidemiological studies do not report about the close relationship between the

\footnotetext{
A. Rothenberger $(\square)$

Child and Adolescent Psychiatry, University of Göttingen,

Von-Siebold Str. 5, 37075 Göttingen, Germany

e-mail: arothen@gwdg.de
}

anxiety/emotions and sleep, since the latter is clinically an important point [5] which is often neglected. A third large-scale epidemiological study comes from Lagos (Nigeria) and reports about psychoactive drugs use among the adolescents-somewhat different frequencies were observed compared to USA and Europe [2]. In a global world, knowing that there exists a large adolescent population in Africa this is worth considering for health politics. Without saying the diagnoses attention-deficit/ hyperactivity disorder (ADHD) and oppositional defiant disorder (ODD) seem to be great implicit problems in these investigated adolescents.

Basic and clinical research in $A D H D$ is the largest scientific field within CAP and is still a driving force for better understanding and treatment of many other child psychiatric problems. This is mainly because of its high frequency of comorbidity.

Hence, many publications on ADHD appear every year and it is sometimes difficult for the usual reader of a journal to understand the meaning of such a paper. Highlighting a paper by experts would help the reader, encourage the researcher and improve the visibility of the journal.

ECAP is a well-known international journal of European origin. ESCAP (European Society for Child and Adolescent Psychiatry) plans to join the journal and declare it the "official organ" of the society. Researchers from all over the world are publishing in ECAP. Not only young researchers especially like it as a platform for their work, but also outstanding scientists submit their papers. For both ADHD and tic disorders, the related European guidelines groups but also other authors have already published numerous articles and supplements in ECAP_-with topics from genetics to clinical aspects. Hence, the flow of information on ADHD is specially a good basis for a research award related to ECAP. 
Therefore, the Editorial Board of ECAP has decided to award a prize for the best ADHD-paper of the year and herewith declares officially the

\section{Announcement of the Research Prize \\ "ECAP-ADHD-Paper of the Year"}

The prize will be awarded each year. The announcement will be published in one of the first issues of ECAP in that year (except the first year, namely 2010, when the selection was done retrospectively). The candidates are the authors of all the papers on ADHD published in ECAP for the year in question. An application by authors is not allowed, but suggestions by the ECAP editorial board are possible. An international jury of experts will select the winner(s) and the prize will be awarded at an European meeting of CAP. In 2011 this is the ESCAP-Congress in Helsinki, where the ECAP-prize for 2010 will be awarded during the opening ceremony.

The decision process of the jury considers mainly the following qualities of a paper: innovation, scientific strength and impact on the future of CAP.

The decision of the jury will be announced in the first ECAP issue of the following year.

The award will have an endowment of 3,000 Euro (in the case of two awards 2,000 Euro each).

The award will be sponsored by Shire Deutschland $\mathrm{GmbH}$.

For the year 2010 eighteen highly informative ADHD related papers appeared in ECAP. This is a good number. The following paper was awarded the research prize:

\section{ECAP-ADHD-Paper of the Year 2010}

Gevensleben H, Holl B, Albrecht B, Schlamp D, Kratz O, Studer P, Rothenberger A, Moll GH, Heinrich H (2010) Neurofeedback training in children with ADHD: 6-month follow-up of a randomised controlled trial. Eur Child Adolesc Psychiatry. 19(9):715-724. [Epub 2010 May 25].

For the Jury, this was clearly the most interesting paper giving new hopes to ADHD children and their families. It follows up a previously reported and internationally well received (e.g. [6]) innovative randomized controlled trial on neurofeedback in ADHD [3] and is important in indicating that the beneficial results of neurofeedback do not disappear once the treatment is discontinued. The expectation that effects would wane has been a reason for not adopting neurofeedback, consequently these results are of substantial clinical interest and encourage the clinicians to make plans, to assess further and offer neurofeedback to their patients on a better evidence base.

However, as Gevensleben et al. [4] stated, Neurofeedback should not replace other forms of behavioural therapy or drug treatment, but should be considered as a relevant option when planning the composition of a multimodal treatment program in ADHD.

We hope that for the ongoing year 2011 many authors will again submit their ADHD Papers to ECAP and I am sure that the jury will again choose an outstanding "ECAP-ADHD-Paper of the Year".

Open Access This article is distributed under the terms of the Creative Commons Attribution Noncommercial License which permits any noncommercial use, distribution, and reproduction in any medium, provided the original author(s) and source are credited.

\section{References}

1. Budisteanu M, Chirieac SM, Tutulan-Cunita AC, Budisteanu B, Arghir A (2011) Novel clinical finding in MECP2 duplication syndrome: a case report. doi:10.1007/s00787-011-0184-2

2. Famuyiwa OO, Aina O, Bankole-Oko O (2011) Epidemiology of psychoactive drug use among adolescents in metropolitan Lagos, Nigeria. doi:10.1007/s00787-011-0180-6

3. Gevensleben H, Holl B, Albrecht B, Vogel C, Schlamp D, Kratz O, Studer P, Rothenberger A, Moll HG, Heinrich H (2009) Is neurofeedback an efficacious treatment for ADHD: a randomised controlled clinical trial. J Child Psychol Psychiatry 50:780-789

4. Gevensleben H, Holl B, Albrecht B, Schlamp D, Kratz O, Studer P, Rothenberger A, Moll GH, Heinrich H (2010) Neurofeedback training in children with ADHD: 6-month follow-up of a randomised controlled trial. Eur Child Adolesc Psychiatry 19:715-724

5. Hansen BJ, Skirbekk B, Oerbeck B, Richter J, Kristensen H (2011) Comparison of sleep problems in children with anxiety and attention deficit/hyperactivity disorders. Eur Child Adolesc Psychiatry 20(6):321-330

6. Pine DS (2009) Editorial: evaluating new and old treatments for ADHD. J Child Psychol Psychiatry 50:767-768

7. Velders FP, Dieleman G, Henrichs J, Jaddoe VWV, Hofman A, Verhulst FC, Hudziak JJ, Tiemeier H (2011) Prenatal and postnatal psychological symptoms of parents and family functioning: the impact on child emotional and behavioural problems. doi:10.1007/ s00787-011-0178-0

8. Wijsbroek S, Hale WW, Raijmakers QAW, Meeus WHJ (2011) The direction of effects between perceived parental behavioral control and psychological control and adolescents' self reported GAD and SAD symptoms. doi:10.1007/s00787-011-0183-3 